\title{
Efecto del género en el cumplimiento del pago de impuestos*
}

\section{Laura Sour**}

Mediante la utilización de evidencia experimental, en este artículo se estudia el efecto del género en el cumplimiento del pago de impuestos, aspecto a menudo ignorado en la evaluación de la incidencia fiscal debido a las dificultades relacionadas con la medición de este comportamiento. Los resultados muestran que al controlar por factores económicos y demográficos, el cumplimiento tributario es mayor en las mujeres que en los hombres. Los resultados del análisis evidencian la necesidad de incluir una perspectiva de género en el diseño de las recomendaciones sobre política fiscal, especialmente en las cuestiones que más afectan a las asalariadas.

Palabras clave: pago de impuestos, experimentos, género, México. Fecha de recepción: 22 de marzo de 2010.

Fecha de aceptación: 13 de febrero de 2014.

\section{Gender Tax Compliance in Mexico}

Using experimental evidence, we examine the effect of gender on tax compliance- an often ignored factor in assessing tax incidence due to the difficulties in measuring its effects. The empirical findings suggest that tax compliance is higher for women than for men, controlling for economic and demographic variables. The results highlight the necessity to take into account gender when designing fiscal policy recommendations, especially those affecting the working women.

Key words: tax compliance, experiments, gender, Mexico.

\section{Introducción}

En el año 2013 la Encuesta Nacional de Ocupación y Empleo (ENOE) de México mostraba una población económicamente activa de 49.6 millones de personas, de las cuales 32.3 millones eran hombres $(62 \%)$

* Traducción del inglés de Graciela Salazar Juárez.

** Profesora e investigadora en la Facultad de Economía y Negocios de la Universidad Anáhuac México Norte. Dirección postal: Av. Universidad Anáhuac núm. 46, col. Lomas Anáhuac, C.P. 52786, Huixquilucan, Estado de México, México. Correos electrónicos: <laura.sour@anahuac.mx>y $<$ laura.sour@gmail.com>.

$\overline{\text { ESTUDIOS DEMOGRÁFICOS Y URBANOS, VOL. 30, NÚM. } 1 \text { (88), 2015, 135-157 }}$ 
y 20 millones mujeres (38\%). La idea general es que las mujeres se han concentrado en "tareas tradicionales de mujeres", como las religiosas o en actividades de voluntariado (Mehra y Gammage, 1999; Fontana, 2003). No obstante, las mujeres mexicanas participan con $51.6 \%$ en el sector de comercio, $47.9 \%$ en el de servicios, $37.6 \%$ en la industria manufacturera, y $26.8 \%$ en otras actividades de la economía. ${ }^{1}$

Si bien la tasa de participación femenina en el mercado laboral en México ha aumentado durante los últimos 60 años, ${ }^{2}$ las mujeres perciben ingresos menores a los de los hombres por actividades similares (Duval y Orraca, 2007; Correía y Katz, 2001; Pagán y Sánchez, 2000; Cerruti y Zenteno, 2000). Tales asimetrías se explican con fundamento en factores tales como sexo, educación, experiencia y discriminación (Oaxaca, 2007).

Un componente a considerar para conocer la calidad de vida de las mujeres que están en el mercado de trabajo formal es su nivel de ingresos; ${ }^{3}$ otro factor igualmente importante es el sesgo implícito de la política fiscal aplicado a las mujeres como contribuyentes, en especial en el caso de México, donde la política tributaria se asume neutral en cuanto a la perspectiva de género: ${ }^{4}$ por mandato constitucional tanto hombres como mujeres deben recibir el mismo trato en el pago de impuestos; en consecuencia, no es posible contar con una tasa de impuestos diferenciada desde un enfoque de género. Algunos dicen

\footnotetext{
${ }^{1}$ Se refiere a la población económicamente activa de 14 años y más. En otras actividades económicas se incluye a personas empleadas en unidades económicas cuya territorialidad no forma parte del país en sentido legal, como es el caso de los trabajadores de la región fronteriza residentes en México, así como personal que labora en embajadas y consulados. Más aún, se consideran también los casos en que no se pudo definir su localidad en términos de la naturaleza que mantiene la unidad económica (INEGI, 2013).

${ }^{2}$ En México ha habido un crecimiento sostenido de las mujeres dentro la población económicamente activa durante los últimos 64 años. De 1950 a 1980 la tasa de crecimiento por década fue de $83 \%$ como promedio. De 1980 a 1990 se registró un leve descenso $(8.1 \%)$. Sin embargo en los siguientes cinco años se observó un aumento de $108 \%$, dado que la cifra de mujeres en la población económicamente activa aumentó de 5644588 en 1990, a 11764720 en 1995. De 1995 a 2013 el aumento registrado fue de $61 \%$ (INEGI, 2013).

${ }^{3}$ Huber (2005) argumenta que en algunos países de América Latina (Argentina, Chile, Costa Rica y Jamaica), cuando la mujer se integra al sector formal tiende a ser contratada por compañías pequeñas, y con frecuencia con un salario menor al de los hombres.

${ }^{4}$ De ninguna manera queremos confundir la variable sexo con el concepto de género. A lo largo de la discusión nos referimos al término "género" porque la literatura respectiva alude a este concepto. No obstante, en el caso del modelo de factores sociodemográficos nos referimos a la variable sexo y edad.
} 
que esta política fiscal, en especial la dirigida a los ingresos, deja de lado la perspectiva de género con el fin de prevenir un efecto discriminatorio. Sin embargo, cuando la política pública aplica el mismo trato fiscal a personas que no son iguales entre sí, las diferencias entre individuos se acentúan aún más (Grown y Valodia, 2007). Por el contrario, si se vincula el género con el pago de impuestos sería posible prevenir los sesgos implícitos que pudiera contener una ley específica en el bienestar de los hombres y de las mujeres (Stotsky, 2005; Álvarez y McCaffery, 2000).

Para entender cabalmente el efecto de la política fiscal desde un enfoque de género (por ejemplo, para apreciar la relación entre la carga fiscal y el sesgo de género que conlleva en determinados países donde la tasa impositiva no difiere por género) es importante estudiar si el cumplimiento en el pago de impuestos es el mismo tanto si proviene de los hombres como de las mujeres. Si las mujeres cumplen con sus obligaciones fiscales con más orden que los hombres, el efecto indirecto del sistema tributario neutro en género es más elevado entre la fuerza de trabajo femenina que en la masculina, debido precisamente a este mayor cumplimiento.

Existen dificultades para llevar a cabo un trabajo empírico sobre el cumplimiento del pago de impuestos, de modo que los experimentos de laboratorio ofrecen un buen punto de partida para investigar las diferencias de género al respecto. Permiten también probar otra variable socioeconómica importante: la edad. Si bien algunos estudios no toman en cuenta esas influencias, existe la tendencia a relacionar una edad más avanzada con la evasión fiscal más baja (véase Torgles y Valve, 2006).

El propósito de esta investigación es probar si el modelo tradicional en el cumplimiento del pago de impuestos es neutral por género y edad al utilizar métodos experimentales y estadísticos. El ejercicio analítico contó con 72 participantes. Las evidencias muestran que las mujeres son más cumplidas en el pago de sus obligaciones tributarias, que la edad es un factor importante, y que una tasa de auditorías más elevada aumenta el cumplimiento de este requisito fiscal. La discrepancia en los niveles de cumplimiento de las obligaciones tributarias entre los hombres y las mujeres es un elemento central en el análisis de la participación y la retención de la fuerza de trabajo femenina dentro del mercado laboral. Si las mujeres reciben un salario menor al de los hombres y si cumplen con sus obligaciones fiscales, entonces se agranda la brecha salarial entre estos grupos. Es decir, las condicio- 
nes del mercado laboral y el sesgo implícito en la política fiscal pueden perpetuar la desigualdad en el bienestar económico y social de las mujeres.

La estructura de este trabajo es la siguiente: en la primera sección se hace una revisión del modelo económico tradicional en el cumplimiento de las obligaciones tributarias; en la segunda se describe el mercado laboral en México, y en la tercera se presentan diversos aspectos sobre la perspectiva de género en el cumplimiento tributario. A continuación se describe la metodología de la economía experimental, y en seguida se explica el diseño experimental. Posteriormente se presentan los resultados, y por último aparecen las conclusiones y las recomendaciones sobre las políticas al respecto.

\section{La perspectiva económica del cumplimiento tributario}

"Cualquier tipo de acción ilegal o intencionada que lleve a cabo un individuo para reducir sus obligaciones tributarias significa evasión de impuestos, tal como la falta de veracidad en su declaración de ingresos, ventas o bienes; sobrestimar la deducibilidad, las exenciones o los créditos, o simplemente las omisiones en su declaración de utilidades" (Alm, 1999: 741). La evasión fiscal provoca que los gobiernos inviertan recursos para impedir que se incurra en la falta de esta obligación, para detectar su magnitud y para castigar a los evasores.

Algunos investigadores han hecho intentos por aislar en sus modelos los matices de motivación e intención subyacentes en las personas cuando llenan las formas de pago para cumplir con los impuestos. En el modelo económico de Michael G. Allingham y Agnar Sandmo (1972), la evasión de impuestos se presenta como un acto intencionado de incumplimiento con la ley tributaria a fin de reducir las obligaciones fiscales. Según este modelo, los contribuyentes deben escoger los ingresos $x$ que van a declarar a las autoridades hacendarias a fin de que puedan maximizar la utilidad esperada. Shlomo Yitzhaki (1974) modificó el modelo Allingham-Sandmo al gravar una penalización a las declaraciones subestimadas, en oposición al ingreso no reportado.

La utilidad para el contribuyente dependerá de su nivel final de ingreso y del ingreso que ocultó a la autoridad hacendaria. La realidad después del impuesto sobre la renta $v$ es igual a $y(l-t)$ donde $t$ es la tasa impositiva uniforme. La probabilidad de ser objeto de auditoría es $p$ y el contribuyente deberá pagar una penalización constante $s$ si evade 
sus impuestos y es objeto de auditoría. El contribuyente debe escoger los ingresos $x$ a declarar a las autoridades hacendarias. Si el contribuyente entra en la categoría de riesgo neutral, su nivel de utilidad se obtiene por:

$$
U^{E}=\operatorname{Max}_{\{x\}}\left\{(1-p) U\left[I_{u}\right]+(p) U\left[I_{D}\right]\right\}
$$

Si el contribuyente decide no declarar y no es objeto de auditoría, su nivel de utilidad será de $I_{u}$.

$$
I_{u}=v+t(y-x)
$$

Cuando el contribuyente decide hacer su declaración, su utilidad será $I_{D}$, o:

$$
I_{D}=v-s t(y-x)
$$

En el supuesto de que los contribuyentes eleven al máximo su utilidad esperada, la primera condición de orden es:

$$
(1-p) U^{\prime}\left[I_{u}\right]-p s U^{\prime}\left[I_{D}\right]=0
$$

Por ende, la ecuación [4] determina el nivel óptimo del ingreso no reportado, dado por $p$ y $s$. El nivel del ingreso reportado será de cero cuando:

$$
p<\frac{1}{(1+s)}
$$

En otras circunstancias nadie evadirá el pago de sus impuestos. En otras palabras, este modelo económico asume que el nivel de cumplimiento lo determina la probabilidad de detectar el fraude así como la aplicación de las multas por incurrir en él. En consecuencia, el modelo puede pronosticar el pago de impuestos cuando es posible detectar el fraude y existe un castigo grave en consecuencia. ${ }^{5}$ Michael

${ }^{5}$ Un riesgo individual neutral es insensible a reportar cualquier nivel de ingreso. Las decisiones individuales óptimas para cualquier objetivo lineal mostrarán un comportamiento de todo o nada, en donde el participante reportará el total de sus ingresos o cero ingresos. Es decir, un individuo de riesgo neutral tiende a exhibir el comportamiento de todo o nada, lo que llevará al aumento de la evasión con una reducción en la tasa de impuesto. 
G. Allingham y Agnar Sandmo reconocen que "para evaluar la eficacia de estas herramientas [infracciones y auditorías] es necesario conocer los estimados empíricos de los efectos vertidos en el estudio" (1972: 338). Hasta el momento no existen conclusiones claras que relacionen buena parte de los factores que determinan el proceso de toma de decisiones de los contribuyentes con el cumplimiento de sus obligaciones. James Andreoni, Brian Erard y Jonathan Feinstein (1998) han enfatizado la necesidad de continuar explorando las diversas influencias sobre el comportamiento de los ciudadanos en torno al cumplimiento de sus obligaciones fiscales.

Las medidas más comunes para evitar la evasión fiscal son las auditorías y las sanciones. Dubin et al. (1990) estimaron el impacto en el impuesto federal sobre la renta por ingresos tributarios en Estados Unidos cuando se incrementa la tasa de auditorías. Concluyen que si se eleva la probabilidad de una auditoría, el ingreso marginal por cobro de impuestos aumentará de manera sustancial, lo que originará un crecimiento considerable de los ingresos tributarios. Según Dubin et al. (1990) los elevados niveles de evasión de impuestos son resultado de revisiones deficientes, pues la tasa de auditorías es baja por la falta de equipo y de personal en las oficinas de la hacienda pública. En consecuencia, si el gobierno aumenta su gasto para hacer valer sus imposiciones, aumentarán los ingresos por tributación. ${ }^{6}$ Sin embargo no siempre la opción de aumentar el número de auditorías es la mejor; tal vez sea más viable aumentar el grado de sanciones, por sus costos más bajos.

\section{El género y el mercado de trabajo en México}

En México las políticas públicas que pretenden lograr la igualdad de género con frecuencia son dirigidas a mujeres indígenas, madres solteras y mujeres desempleadas mediante programas que benefician a estos grupos considerados como los más vulnerables. Si bien estos

${ }^{6}$ Existen otros factores, además de las medidas restrictivas, que pueden explicar también los bajos niveles en el cumplimiento: $i$ ) falta de honestidad, ii) la apreciación de que el sistema tributario es injusto, iii) la idea de que el número de individuos en la economía que pagan sus impuestos es bajo, iv) el considerar que el gobierno malgasta el dinero de los contribuyentes, y $v$ ) el creer que el marco regulatorio es complejo y no transparente. Sin embargo, en este trabajo únicamente se discute el efecto restrictivo del gasto por género. Para una revisión completa de la literatura, véase Andreoni et al., 1998. 
grupos sociales son importantes, existen otros cuya vulnerabilidad es más difícil de reconocer. Tal es el caso de las mujeres trabajadoras. De la población con mayores ingresos en el país (más de 5 salarios mínimos) la vasta mayoría corresponde a hombres $(69 \%)$, y más de la mitad de la población con menores ingresos son mujeres (INEGI, 2013).

Correía y Katz (2001) nos informan que en el año 2000 el 37\% de las mujeres estaba empleada o buscaba un empleo, en comparación con el $79 \%$ de los hombres. Esta brecha se amplía cuando se toma en cuenta el estado civil: $93 \%$ de los hombres casados contaba con trabajo, pero sólo lo tenía 21\% de las casadas. La incidencia se mantiene en el sector de trabajadores independientes (Pagán y Sánchez, 2000). Para el año 2013, el porcentaje de la población económicamente activa (empleada o desempleada) era de $43 \%$ para las mujeres y de $77 \%$ para los hombres. Si se considera el estado civil el $68 \%$ de hombres casados tenía un trabajo, en comparación con el 52\% de las casadas. En resumen, las cifras porcentuales de la población masculina económicamente activa han disminuido, mientras que las cifras de la población femenina económicamente activa han aumentado, tal como sucedió con las casadas (INEGI, 2013).

Otro contraste importante se relaciona con los salarios. En 1987 las mujeres en México percibían ingresos salariales cerca de $20.8 \%$ menores que los hombres. Esta diferencia aumentó dos puntos porcentuales en 1993 (Brown, Pagán y Rodríguez-Oreggia, 1999). Pero Correía y Katz (2001) apuntan que las mujeres perciben entre 36 y $50 \%$ del ingreso que reciben los hombres por realizar las mismas actividades. Estas cifras son similares a las encontradas en 18 países de América Latina, donde los hombres perciben entre 9 y $27 \%$ más que las mujeres (Hoyos y Ñopo, 2010). Lo anterior muestra la necesidad en México de crear mecanismos fiscales que no aumenten la disparidad de las condiciones económicas y laborales de la población femenina.

Pagán y Sánchez (2000) argumentan que el género es el factor más importante para explicar la diferencia en los patrones de participación en el mercado laboral de los hombres y las mujeres. Según Oaxaca (2007), la diferencia salarial por género que no puede justificarse mediante variables perceptibles tales como la educación, la experiencia y la productividad, es atribuible a la discriminación. Martínez y Acevedo (2004) y Brown, Pagán y Rodríguez-Oreggia (1999) comprobaron que las diferencias porcentuales en los salarios de las mujeres no tenían explicación por los incentivos iniciales. Popli (2012) encontró que la brecha salarial por género es más elevada en el sector 
formal que en el informal, y Frías (2011) argumenta que las mujeres que laboran en oficinas gubernamentales son proclives a sufrir acoso sexual y a padecer prácticas de discriminación por embarazarse.

\section{Género e impuestos}

La política fiscal puede reducir o aumentar la brecha de género en la medida en que la remuneración salarial determina la entrada y participación continua de las mujeres en el mercado laboral. Por lo tanto, resulta importante investigar el enlace entre el género y la incidencia fiscal porque arrojará algunas ideas sobre los sesgos implícitos o explícitos que puede generar una ley específica en la materia sobre el bienestar de hombres y mujeres.

Analizar el sesgo en favor de un género en relación con los impuestos es relativamente reciente. Suden y Surette (1998) y Deere y Doss (2006) encontraron significativas diferencias de género en el monto del ahorro para el retiro en Estados Unidos. Es más, las mujeres tienden a gastar una proporción mayor de su ingreso en comida, educación y cuidado de la salud (Basu, 2006). Este mismo patrón se reporta en el caso de México: "las mujeres de la clase media mexicana tienden a gastar más en la manutención de sus hogares" (Cerruti y Zenteno, 2000: 69).

La literatura y los datos sugieren que las autoridades hacendarias deberían examinar los efectos de los impuestos, tanto explícitos como implícitos, en hombres y mujeres y reconocer que ellos y ellas tienen diferente propensión al ahorro y al consumo. En consecuencia, cualquier cambio en los precios relativos de estos productos, por cambios en las políticas fiscales, pueden afectar la decisión de las mujeres en una dimensión distinta a la de los hombres.

$\mathrm{Si}$ en México, como en los países en desarrollo, los patrones de consumo difieren por género y las utilidades que recibe la fuerza de trabajo femenina son inferiores a la de los hombres por actividades similares (como muestran las evidencias), poner en marcha una tasa impositiva igualitaria generará una inclinación negativa en el bienestar femenino. Hasta la fecha los análisis se han enfocado en buscar las diferencias de género en la asignación de los recursos monetarios (patrones de consumo), pero no se ha estudiado si prevalecen estas diferencias cuando los individuos toman la decisión de cumplir o no con sus obligaciones fiscales. La población femenina en México tiene 
un papel importante en áreas donde los impuestos no se recaudan automáticamente, como el comercio y la industria manufacturera: las mujeres contribuyen con $51.6 \%$ en el sector de comercio; $47.9 \%$ en el de servicios; $37.6 \%$ en la industria de manufactura, y $26.8 \%$ en otras actividades económicas (INEGI, 2013). Se trata de un aspecto importante en especial en el caso de las mujeres que pertenecen al régimen de honorarios y de negocios, pero también afecta a las asalariadas, cuya permanencia en el mercado laboral es inestable y no cuentan con perspectivas de largo plazo.

Álvarez y McCaffery (2000) reportan diferencias de género en las actitudes respecto al pago de impuestos en las economías desarrolladas. Barnett y Grown (2004) desarrollaron una tipología de impuestos y género e incluyeron el género y otras variables socioeconómicas ignoradas en otros modelos económicos, tales como la actividad económica y el nivel de desarrollo del país. Baldry (1987), al utilizar el análisis experimental, encontró que las mujeres cumplen más que los hombres con el pago de sus impuestos. Fallan (1999) especula que las diferencias encontradas en las actitudes respecto al pago de impuestos entre los hombres y las mujeres pueden estar influidas por un conocimiento diferente de las obligaciones fiscales; el autor concluye que el género tiene un efecto de predicción porque explica los cambios de actitud hacia las cuestiones éticas de los impuestos, pero ello no afecta la percepción de la justicia tributaria. Si bien esa investigación constata las diferencias de género en el cumplimiento del pago de impuestos, deja abierta la pregunta sobre los factores que puedan explicar dichas diferencias. Martínez y Acevedo (2004) han hecho esfuerzos por explicar esas asimetrías, apoyados en elementos tales como el género, la educación y la experiencia. Sin embargo aún queda un componente de esta diferencia atribuible a la discriminación. Uno de los siguientes pasos también deberá incluir en el análisis escenarios en donde se controle la aversión al riesgo entre hombres y mujeres.

La falta de evidencia sobre la diferencia de género (por sexo del contribuyente) en el cumplimiento de las obligaciones fiscales hace que los métodos de laboratorio sean una buena herramienta para comenzar a investigar el comportamiento entre hombres y mujeres, y determinar si la diferencia de género afecta el cumplimiento en el pago de impuestos en México y en qué magnitud. ${ }^{7}$

${ }^{7}$ En este sentido, Estados Unidos es el único caso en el que dicho asunto se ha analizado extensamente. La evidencia empírica más confiable sobre el pago de impuestos en ese país es el Taxpayer Compliance Measure Program (TCMP) [Programa de 


\section{Economía experimental}

La falta de información empírica "dura" sobre el cumplimiento de las obligaciones fiscales ha obligado a los investigadores a generar su propia información mediante encuestas de contribuyentes o simulaciones experimentales de evaluaciones del impuesto sobre la renta. En estas encuestas se solicita a los contribuyentes que reporten el pago de sus impuestos a tiempo, o que realicen cualquier otra actividad de la cual se pueda inferir tal cumplimiento. Sin embargo es difícil descifrar la información porque se requiere de total honestidad y una memoria perfecta si se desea que la respuesta refleje las actividades de interés. Un método alternativo de generar información requiere del diseño de un experimento que replique el cumplimiento tributario, y que los individuos tengan el mismo tipo de incentivos para declarar sus impuestos, como si se tratara del proceso real. Este tipo de experimentos consiste por lo general en un juego con participantes que hacen sus declaraciones, pagan sus impuestos, son sujetos de revisiones (auditorías) y pagan las multas en caso de que se detecte que no cumplieron con sus obligaciones tributarias en secuencias de periodos múltiples.

El método experimental resulta muy efectivo para probar pronósticos teóricos. Este experimento excluye las variables irrelevantes, genera información de especial importancia para el análisis, y puede diseñarse a modo de que determine las variables que son endógenas y las que son exógenas, sin ambigüedades. También evita problemas de multicolinealidad en las variables independientes.

Sin embargo, el enfoque experimental tiene algunos problemas evidentes: la magnitud de las ganancias y las pérdidas de los sujetos en el cumplimiento de sus obligaciones puede ser diferente de aquellas asociadas con las decisiones reales; también los participantes en el experimento pueden actuar de manera distinta a la actividad económica real en el cumplimiento tributario. No obstante, no se puede inferir de manera automática que los participantes se comportarán en

\footnotetext{
Medición del Contribuyente], del Internal Revenue Service (IRS) [Servicio de Tributación Nacional]. El TCMP supervisa constantemente las utilidades de cada contribuyente mediante una muestra aleatoria; a pesar de que tiene fallas en detectar algunas fuentes de ingreso que no es necesario reportar (como las de los trabajadores independientes y las de quienes reciben en efectivo la mayoría de sus ingresos), se le reconoce como la mejor información "dura" disponible para las economías desarrolladas. Para conocer algunos estudios internacionales, véase Graetz y Wilde, 1985; sobre Jamaica: Alm, Bahl y Murray, 1990; sobre los Países Bajos: Hessing et al., 1992, y sobre España: De Juan, Lasheras y Mayo, 1994.
} 
el experimento de manera diferente de su comportamiento real en el cumplimiento de obligaciones, a pesar de que muchos individuos pueden tomarlo como juego. Si los resultados experimentales son consistentes con los que derivaron de la información "dura", los primeros resultan de utilidad pues se trata de una representación favorable del proceso real.

Una característica importante del diseño experimental es que las instrucciones utilizan terminología "neutra". Es decir, las referencias relativas a impuestos, auditorías, ingresos declarados y similares, son sustituidas por palabras neutras tales como pagos, cheques, ingresos reportados, etc. En un paradigma experimental un principio básico es que en las instrucciones se utilicen términos neutros para evitar los efectos de contexto y el desvío de las elecciones pronosticables (para conocer una discusión más amplia sobre el diseño experimental véase Plott, 1987 y Smith, 1982; también consúltese Guala, 2005). En investigaciones previas al respecto se ha inducido inadecuadamente la preferencia del participante porque se le han dado instrucciones que colocan al experimento en un contexto de evasión de impuestos; es decir, la terminología utilizada en dichos trabajos previos pudo haber proporcionado un contexto no deseado en el experimento, y las decisiones de los participantes pudieron haber reflejado los valores que asociaron con términos como "cumplimiento fiscal" o "evasión fiscal", más que con las recompensas o las multas que enfrentaron en el experimento per se (por ejemplo, véase Bosco y Mittone, 1997, y Friedland, Maital y Rutenberg, 1978).

Al diseñar el experimento debe ponerse atención en que los participantes otorguen la información completa de la situación que enfrentarán en el laboratorio; tomarán sus decisiones únicamente con las bases que se les otorguen, tales como pagos, recompensas e instrucciones. ${ }^{8}$ En este caso se colocaron pliegos de cartón delgado alrededor de cada una de las terminales de computadora para evitar que los participantes vieran la terminal contigua y no se respetara la privacidad de cada uno de ellos.

${ }^{8}$ Vernon L. Smith (1982) ha identificado cuatro condiciones que son suficientes para desarrollar un sistema microeconómico de laboratorio: $i$ ) insaciabilidad (los sujetos prefieren más que menos); ii) prominencia (las decisiones de los sujetos tienen efectos elevados en recompensas); iii) dominancia (la estructura de recompensas está por encima de cualquier costo asociado con la participación en las actividades del experimento); y $i v$ ) privacidad (a cada sujeto en un experimento se le provee información únicamente sobre su propias alternativas de utilidades). 


\section{Diseño experimental}

La economía experimental incluye la creación de un sistema macroeconómico de laboratorio. La esencia de tal sistema es controlar las opciones que los participantes pueden elegir y así poder capturar los matices que contengan los reportes voluntarios de ingreso. El objetivo principal del experimento es examinar si la elevada probabilidad de una auditoria y una penalidad son las causas para que se paguen los impuestos, y además determinar si existe el efecto del género en el cumplimiento del pago de impuestos en un ambiente controlado dentro de un sistema tributario muy simple.

Para conocer el efecto del género en el cumplimiento del pago de impuestos en un ambiente de laboratorio controlado, se asignó a los participantes un ingreso seleccionado de manera aleatoria por la computadora, y éstos decidieron qué tanto impuesto debían reportar. Cuando declaran voluntariamente sus ingresos, pagan los impuestos bajo una tasa específica; pero también pueden optar por no pagarlos o reportar menos. Saben que existe una probabilidad fija de auditoría, y que si se les detecta alguna trampa deberán pagar una penalización por no pagar impuestos. Por ejemplo, la tasa de multa de 2 implica que los sujetos pagarán los impuestos que deben, más una penalización equivalente al total de éstos. Cuando se completa una ronda los sujetos aparecen individualmente con sus nuevos saldos. Este proceso se repetirá por un número fijo de rondas, pero los participantes no sabrán el total de éstas a fin de evitar los efectos finales del tratamiento. Cada sesión se repitió 30 veces. El propósito de cada ronda es el de representar un año fiscal.

El experimento fue conducido en un laboratorio donde los cálculos se hicieron por computadora; consta de nueve sesiones, cada una de ellas subdividida en tres partes. Después de las primeras diez rondas (parte 1), los participantes enfrentan otro nivel de parámetro para las siguientes diez. Entonces se compara el cumplimiento en presencia y en ausencia de ese cambio específico. Las sesiones 1 a 3 examinan el efecto de tres niveles diferentes de probabilidad de auditoría: 5, 30 y $50 \%$. Las sesiones 4 a 6 cambian la tasa de castigo de los impuestos no reportados del nivel 2 al 4 y del nivel 6 para terminar, pero queda constante la probabilidad de una auditoría así como la tasa impositiva. Las tres últimas sesiones capturan la respuesta de la tasa de cumplimiento a los cambios en la tasa de impuesto (10, 30 y 40\%). Los participantes están organizados en grupos de ocho personas y a cada uno de ellos se le ha asignado un número de identificación. 
Antes del inicio de cada sesión la computadora da una variación de ingreso de 25 a 200 fichas a cada participante, quienes leerán su propia hoja de instrucciones y la guardarán consigo como referencia durante el experimento. ${ }^{9}$ En el cuadro 1 se presenta el diseño experimental.

En el diseño se escogieron algunos parámetros de valor para aproximarlos a los valores reales que enfrentan las personas en México. Por ejemplo, una tasa de impuesto del $30 \%$ en el experimento es comparable a la que enfrentan los contribuyentes en el grupo de impuesto marginal alto. Es importante recordar que uno de los objetivos principales de este trabajo es probar, en un ambiente controlado, las predicciones teóricas y observar las simetrías no predecibles. De esta forma, las predicciones se interpretan en relación con la teoría. En consecuencia, en el experimento son centrales los parámetros de valor para generar la serie de predicciones en términos del comportamiento observado.

Los 72 participantes en el experimento son alumnos voluntarios, estudiantes de licenciatura del Centro de Investigación y Docencia Económicas (CIDE) de la Ciudad de México: 47\% mujeres y 53\% hombres. Casi $60 \%$ de ellos tenía experiencia laboral. Se les informó que se pagaría a cada uno según su comportamiento. ${ }^{10} \mathrm{El}$ equivalente era de 50 fichas por un peso mexicano.

Incluir a estudiantes es una práctica casi universal en la economía experimental; la disponibilidad y el costo tienen un papel específico en este ejercicio. Más aún, los experimentos diseñados de manera adecuada evalúan la decisión personal e individual, y no existe razón para poner en duda que los estudiantes tienen acceso a los mismos procesos cognitivos que quienes no son estudiantes. En los trabajos que comparan estudiantes con quienes no lo son, no se han encontrado diferencias significativas (Plott, 1987; Guala, 2005). Es también interés de este trabajo probar la teoría que supone que los agentes están motivados a participar únicamente con el fin de maximizar la utilidad esperada. Una muestra con agentes no susceptibles de saciarse con el dinero, como estudiantes de licenciatura, es suficiente para sacar adelante el experimento (Smith, 1982: 937).

\footnotetext{
${ }^{9}$ Las instrucciones fueron escritas originalmente en idioma español.

${ }^{10}$ Ingresos individuales con un rango de entre siete y once dólares dependiendo de la actuación del participante en el experimento.
} 


\section{CUADRO 1}

\section{Diseño experimental}

\begin{tabular}{clcl}
\hline Sesión & Tasa de auditoría & Tasa de multas & Tasa de impuesto \\
\hline 1 & $5 \%, 30 \%, 50 \%$ & 2 & $30 \%$ \\
2 & $30 \%, 50 \%, 5 \%$ & 2 & $30 \%$ \\
3 & $50 \%, 5 \%, 30 \%$ & 2 & $30 \%$ \\
4 & $30 \%$ & $2,4,6$ & $30 \%$ \\
5 & $30 \%$ & $4,6,2$ & $30 \%$ \\
6 & $30 \%$ & $6,2,4$ & $30 \%$ \\
7 & $30 \%$ & 2 & $10 \%, 30 \%, 40 \%$ \\
8 & $30 \%$ & 2 & $30 \%, 40 \%, 10 \%$ \\
9 & $30 \%$ & 2 & $40 \%, 10 \%, 30 \%$ \\
\hline
\end{tabular}

\section{Resultados}

El análisis de los resultados experimentales tiene como base los ingresos declarados para cada tratamiento; en consecuencia esta variable está truncada en cero. Para organizar la estructura de la variable dependiente se utilizó el modelo Tobit y calcular así los coeficientes de las variables independientes.

El modelo económico indica que una probabilidad más elevada de auditoría y multa origina que las personas cumplan con sus obligaciones fiscales. Por lo tanto, el porcentaje de cambios del ingreso declarado respecto de un cambio porcentual en la tasa de auditoría o en la tasa de castigo nos debería indicar por qué las personas pagan sus impuestos. Es más, el experimento también mostró la influencia del efecto del género en el cumplimiento de sus contribuciones; la edad, otra variable sociodemográfica incluida, también muestra resultados importantes en esta obligación (véase el cuadro 2).

Primero, los resultados muestran que un aumento en la tasa de auditoría puede provocar el incremento en los ingresos tributarios. Este resultado confirma las predicciones del modelo de AllinghamSandmo, que es consistente también con el trabajo empírico de Dublin, Graetz y Wilde (1990).

Segundo, si bien los bajos costos de administración que genera el aumentar las tasas de penalización hacen que esta política sea popular entre la mayoría de las autoridades fiscales, los resultados estimados 
Sour, EFECTO DEL GÉNERO EN EL CUMPLIMIENTO DEL PAGO

CUADRO 2

Estimado Tobit. Variables dependientes del ingreso declarado*

\begin{tabular}{lcc}
\hline & Coeficiente & Elasticidad \\
\hline Constante & 7.296 & - \\
& $(3.41)$ & 1.1172 \\
Ingreso & 0.548 & \\
& $(0.02)$ & 0.373 \\
Tasa de auditoría & 6.389 & \\
& $(1.08)$ & -0.0082 \\
Tasa de multas & 0.156 & \\
& $(0.09)$ & -0.2872 \\
Tasa de impuesto & -5.016 & -1.704 \\
& $(1.55)$ & \\
Edad & -.437 & - \\
Género & $(0.10)$ & \\
& 0.418 & \\
N & $(0.24)$ & \\
Log-probabilidad & 2160 & \\
LR & -5901.49 & \\
\end{tabular}

*La variable dependiente equivale al ingreso declarado. Cada elasticidad se ha calculado en valores promedio de las variables. Los valores de márgenes de error se muestran entre paréntesis.

muestran que el coeficiente es cercano a cero y sin importancia. Este hecho es consistente con la mayoría de las reflexiones en la literatura sobre el tema, en la que se asegura que es poca la evidencia en cuanto a que los castigos severos reducen el incumplimiento de las obligaciones fiscales (Doob y Webster, 2003). ${ }^{11}$

${ }^{11}$ La evidencia indica que las tasas más elevadas de auditorías -no las multas más elevadas- aumentan el cumplimiento. Las autoridades hacendarias deben tomar en cuenta los costos de administración por aumentar la probabilidad de realizar auditorías al diseñar las políticas impositivas. Por ejemplo, los resultados sugieren que la oficina encargada de estas políticas puede aumentar el pago de impuestos al cambiar la estrategia impositiva, aunque existen límites a las estrategias que se basan únicamente en más imposiciones. En particular, si el impacto es no lineal, el efecto disuasivo en una tasa de auditoría elevada disminuirá en el tiempo (Alm et al., 1992). En consecuencia, quienes diseñan las políticas deben interpretar los resultados experimentales con cautela. 
Tercero, las tasas de cumplimiento descienden cuando aumenta la tasa tributaria. El resultado refuta los hallazgos teóricos del modelo de Shlomo Yitzhaki (1974), pero es consistente con diversos estudios empíricos que apoyan la idea de que el ahorro en impuestos derivado del incumplimiento de las obligaciones fiscales es una función de la tasa tributaria (Clotfelter, 1983; Slemrod, 1985; Crane y Nourzad, 1986; Bladry, 1987; Poterba, 1987, y Friedland, Maital y Rutenberg, 1978). En consecuencia, los incentivos para el incumplimiento aumentan en la medida en que se eleva la tasa tributaria.

El resultado más importante del experimento es que las variables sociodemográficas, tales como la edad y el sexo, son determinantes significativos del cumplimiento de pago de impuestos en México. Si bien no existe una variación importante en la edad de los estudiantes que participaron en el experimento, en el caso de México la edad puede cambiar este cumplimiento de manera negativa y su efecto es más profundo que el efecto positivo de una auditoría. ${ }^{12}$ Este resultado refuerza los hallazgos de Friedland et al. (1978), pero va en contra de los de Baldry (1987). Esta situación es especialmente importante en México, donde los jóvenes representan la mayoría de la población. Entonces, a medida que los individuos van envejeciendo, su tasa de cumplimiento puede ir a la baja. Es importante mencionar también que el género es una variable sociodemográfica muy significativa, más que la tasa de auditoría, que una variable económica. Una posible explicación sobre este resultado es que la decisión de los contribuyentes tiene como base la probabilidad de detección y penalidad. En consecuencia, la diferencia de sexo en la animadversión al riesgo puede ser un factor importante para explicar empíricamente la tasa de cumplimiento fiscal (Eckel y Grossman, 2005).

\section{Conclusiones}

Examinamos algunos de los posibles determinantes económicos del cumplimiento en el pago de impuestos personales en México tomando como base la información experimental, a modo de compensar la falta de investigación empírica sobre este problema. La intención de nuestro estudio fue probar si el modelo económico aplicado para el cumplimiento de las obligaciones fiscales es neutral en el caso de Mé-

\footnotetext{
${ }^{12} \mathrm{El}$ coeficiente es importante al nivel de 10 por ciento.
} 
xico. La lectura más importante es que las mujeres son más cumplidas con sus contribuciones, y que la edad puede cambiar negativamente este cumplimiento.

En este sentido es importante analizar las razones de quienes no favorecen que se incorpore una perspectiva de género en la política fiscal. Los resultados demuestran la necesidad de tomar en cuenta el enfoque de género al diseñar las recomendaciones de una política tributaria. Por consiguiente, los asesores fiscales deben examinar la incidencia de la tributación desde el punto de vista de las mujeres y desde el de los hombres, y reconocer que tienen diferente propensión a cumplir con dichas obligaciones. Sin embargo, y aunque por mandato constitucional hasta la fecha no es posible modificar el pago de impuestos para reconocer estas diferencias, es recomendable analizar ciertas propuestas en relación con su efecto en la entrada y participación de las mujeres en el mercado laboral. Es muy cierto que en México las mujeres tienden a gastar más en la manutención de sus hogares (Cerruti y Zenteno, 2000: 69), por lo que debería considerarse la posibilidad de incluir exenciones en gastos de educación y cuidado a personas mayores.

Además de la detección y el castigo, en el caso de México existen otros factores que influyen en la decisión de los contribuyentes y que complementan la evaluación de la incidencia fiscal por género. Los resultados muestran la necesidad de fomentar la discusión y analizar la posibilidad de que las políticas gubernamentales incorporen exenciones fiscales desde la perspectiva de género, a fin de mejorar la remuneración de las mujeres y aumentar su estabilidad en el mercado laboral para así alcanzar una igualdad de género.

Para entender mejor las diferencias de género en el mercado laboral de México es necesario realizar en el futuro investigaciones sobre la forma en que la estructura del sistema fiscal puede influenciar la decisión de las mujeres a participar en el mercado laboral y en la remuneración de las horas trabajadas en el mercado formal. El siguiente paso sería analizar si la influencia de las normas sociales explica el pago de impuestos por género (sexo de los contribuyentes); tanto sociólogos como psicólogos podrían aportar análisis más satisfactorios sobre este fenómeno.

La evidencia aquí presentada puede contribuir a la discusión de los temas de género en la literatura sobre análisis fiscal, dado que las políticas tributarias como las del gasto afectan el ingreso y la igualdad de género. Si las mujeres cumplen cabalmente con sus obligaciones 
fiscales y existen diferencias de género en el impacto de los impuestos en la oferta laboral, entonces una política fiscal diferenciada entre hombres y mujeres podría igualar las disparidades de género en el país.

Por último, los experimentos permiten analizar el efecto de factores que no son objeto de estudio en el trabajo teórico; también generan información desde diversos marcos en donde hay control sobre las influencias externas. Por consiguiente, los estudios experimentales pueden contribuir a dar a conocer nuestra interpretación de que los factores fiscales afectan la equidad de género.

\section{APÉNDICE}

\section{Instrucciones}

Éste es un experimento sobre la economía de la toma de decisiones. Las instrucciones son sencillas y si las sigues detenidamente, tendrás oportunidad de ganar una cantidad considerable de dinero que se te pagará en efectivo al final de la sesión.

Los participantes están divididos en grupos de ocho personas. Cada grupo contará con las mismas ocho personas durante toda la sesión. No se te revelará la identidad de los demás miembros de tu grupo. Está prohibido comunicarse con cualquiera de las demás personas durante la sesión. Si no acatas las reglas nos veremos obligados a excluirte del experimento y no recibirás ningún pago.

La sesión consta de varias rondas, cada una de las cuales es independiente de las demás. En cada ronda se te pedirá que tomes una decisión, y el conjunto de estas decisiones determinará tus ingresos totales. No tendrás conocimiento del número total de rondas. Al inicio de cada sesión cada participante recibirá 2000 fichas. Tendrás la oportunidad de hacer crecer esta cantidad en cada ronda. Al final de la sesión podrás canjear las fichas que hayas acumulado a un tipo de cambio de un peso por cada 50 fichas. Por ejemplo, si al concluir el experimento tienes un saldo de 5000 fichas en la computadora, recibirás 100 pesos. Siéntete con toda libertad para intentar ganar todo el dinero que puedas.

Al inicio de cada ronda, en la esquina superior izquierda de la pantalla de la computadora aparecerán el número de la sesión, del participante y de la ronda. En cada ronda recibirás una nueva cantidad de fichas. Las cantidades exactas que tú y las demás personas de tu 
grupo reciban se definirán aleatoriamente por computadora y serán de entre 25 y 200 fichas, en incrementos de 25 . Todas las cantidades tienen la misma probabilidad de ser asignadas y sólo tú sabrás la cantidad que recibiste. Tienes la opción de conservar tus fichas o de reportarlas en el campo de captura "Fichas reportadas". Puedes destapar cualquier cantidad de fichas desde cero hasta el número de fichas que realmente recibas.

Pagarás el 30\% de las fichas que reportes. Por ejemplo, si recibes 100 fichas y destapas 70, pagarás 21 fichas (0.3 multiplicado por 70). No pagarás nada por el dinero (fichas) que no destapes y sólo tú sabrás la verdadera cantidad de dinero que recibas al inicio de cada ronda. Una vez que hayas decidido el número de fichas que quieres destapar, deberás anotar este número en la hoja de reporte (hoja amarilla), así como el número de la ronda. En el ejemplo anterior, capturarías los siguientes números en la hoja de reporte:

\begin{tabular}{|c|c|}
\hline Ronda & Fichas destapadas \\
\hline 1 & 70 \\
\hline
\end{tabular}

Ahora, espera a que se te indique que oprimas el botón "Aceptar". Por favor revisa el número de fichas que optes por reportar, ya que no podrás cambiar tu reporte una vez que hayas oprimido el botón "Aceptar". Cuando todos hayan reportado sus fichas, algunos participantes podrán ser seleccionados para una revisión. En esta revisión, la computadora comparará el número exacto de fichas que tiene la persona en la ronda en curso contra los niveles reportados. Si se te hace una revisión, se descubrirán todas las fichas que hayas recibido y no reportado. Pagarás la diferencia (el 30\% de las fichas recibidas y no reportadas) más una penalización. En esta sesión, pagarías la diferencia más otro monto por la misma cantidad. En el ejemplo anterior, pagarías 18 fichas adicionales, es decir, la diferencia ( 30 fichas multiplicado por 0.3), más una sanción de 9 fichas. La computadora calculará el pago de la diferencia y lo restará de tu saldo. Sólo tú sabrás el resultado de tu propia revisión.

El procedimiento para seleccionar a una persona para revisión es el siguiente: cada persona tiene un número de identificación, entre 1 y 8 , que aparece en su pantalla. La tómbola que aparece en la esqui- 
na superior derecha de tu pantalla contiene bolas numeradas del 1 al 20. Después de que todos hayan destapado sus fichas, se sacará una bola de la tómbola. Si el número de la bola que se saque es del 1 al 8 , la persona que tenga ese número de identificación será sujeta a revisión. Si el número es del 9 al 20, no se revisará a nadie en esa ronda. Una vez que se haya sacado la bola de la tómbola, espera a que se te pida hacer clic en el botón “¿Desea continuar?”. Podrás continuar con la siguiente ronda después de hacer clic en el botón indicado.

Comenzaremos con dos rondas de práctica para que te familiarices con el proceso de pago, destape y revisión. Estas rondas de práctica no se contarán en el cálculo de tu pago. Al final de las dos rondas de práctica, tu saldo se restablecerá en 2000 fichas antes de iniciar las rondas reales.

¿Tienes alguna pregunta? Por favor levanta la mano. No hagas ninguna pregunta en voz alta.

Cuando termines de leer estas instrucciones por favor colócalas boca abajo en el escritorio.

\section{Bibliografía}

Andreoni, James, Brian Erard y Jonathan Feinstein (1998), “Tax Compliance”, Journal of Economic Literature, núm. 36, pp. 818-860.

Allingham, Michael G. y Agnar Sandmo (1972), "Income Tax Evasion: A Theoretical Analysis”, Journal of Public Economics, núm. 1, pp. 323-338.

Alm, James (1999), "Tax Compliance and Administration”, en W. Bartley Hildreth y James A. Richardson (coords.), Handbook on Taxation, Nueva York, Marcel Dekker, pp. 741-768.

Alm, James, Roy Bahl y Matthew N. Murray (1990), “Tax Structure and Tax Compliance", The Review of Economics and Statistics, vol. 72, núm. 4, pp. 603-613.

Alm, James, Betty Jackson y Michael McKee (1992), "Estimating the Determinants of Taxpayer Compliance with Experimental Data”, National Tax Journal, vol. 45, núm. 1, pp. 107-114.

Álvarez, R. Michael y Edward J. McCaffery (1999), "Gender and Tax", Olin Working Paper, vol. 99, núm. 11, Los Ángeles, USC Law School, pp. 1-30.

Álvarez, R. Michael y Edward J. McCaffery (2000), "Is there a Gender Gap in Fiscal Political Preferences?", Law and Economics Research Paper Series, núm. 00-5, Los Ángeles, USC Law School, pp. 1-23.

Baldry, Jonathan C. (1987), "Income Tax Evasion and the Tax Schedule: Some Experimental Results”, Public Finance, vol. 422, pp. 357-383. 
Barnett, Kathleen y Caren Grown (2004), Gender Impacts of Government Revenue Collection: The Case of Taxation, Londres, Commonwealth Secretariat.

Basu, Kaushik (2006), "Gender and Say: A Model of Household Behaviour with Endogenously Determined Balance of Power”, The Economic Journal, vol. 116, núm. 511, pp. 558-580.

Bosco, Luigi y Luigi Mittone (1997), "Tax Evasion and Moral Constraints: Some Experimental Evidence”, Kyklos, vol. 50, núm. 3, pp. 297-324.

Brown, Cynthia, J., José A. Pagán y Eduardo Rodríguez-Oreggia (1999), “Occupational Attainment and Gender Earnings Differentials in Mexico", Industrial and Labor Relations Review, vol. 53, pp. 123-135.

Cerrutti, Marcela y René Zenteno (2000), “Cambios en el papel económico de las mujeres entre las parejas mexicanas”, Estudios Demográficos y Urbanos, vol. 15, núm. 1 (43), pp. 65-95. Disponible en: <http://www.redalyc.org/ articulo.oa?id=31204304 $>$ y $<$ http: / jstor.org/stable /40315022> (consulta: enero de 2010).

Clotfelter, Charles T. (1983), "Tax Evasion and Tax Rates: An Analysis of Individual Returns”, The Review of Economics and Statistics, vol. 65, pp. 363-373.

Correía, María y Elizabeth Katz (2001), "The Economics of Gender in Mexico: Work, Family, State, and Market”, en Marcelo Giugale, Oliver Lafourcade y Vinh Nguyen (coords.), Mexico: A Comprehensive Development Agenda, Washington, World Bank, pp. 553-568.

Crane, Steven E. y Farrokh Nourzad (1986), "Inflation and Tax Evasion: An Empirical Analysis", The Review of Economics and Statistics, vol. 68, pp. 217-223.

Deere, Carmen Diana y Cheryl Doss (2006), "The Gender Asset Gap: What Do We Know and Why Does It Matter?”, Feminist Economics, vol. 12, núms. 1-2, pp. 1-50.

Doob, Anthony y Cheryl M. Webster (2003), "Sentence Severity and Crime: Accepting the Null Hypothesis", Crime and Justice: A Review of Research, vol. 30, pp. 143-195.

Dublin, Jeffrey A., Michael J. Graetz y Louis L. Wilde (1990), "The Effects of Audit Rates on the Federal Income Tax 1977-1986”, National Tax Journal, vol. 90, núm. 43, pp. 395-409.

Duval, Robert y Pedro Orraca (2007), "A Cohort Analysis of Labor Participation in Mexico 1987-2008”, CIDE Working Paper, núm. 440, México, CIDE, pp. 1- 34.

Eckel, Catherine C. y PhilipJ. Grossman (2005), "Men, Women and Risk Aversion: Experimental Evidence”, en Charles R. Plott y Vernon L. Smith (coords.), Handbook of Results in ExperimentalEconomics, Nueva York, Elsevier.

Fallan, Lars (1999), "Gender, Exposure to Tax Knowledge, and Attitudes Towards Taxation; An Experimental Approach”, Journal of Business Ethics, vol. 18, núm. 2, pp. 173-184.

Fontana, Marcia (2003), "The Gender Effects of Trade Liberalization in Developing Countries: A Review of the Literature", Discussion Papers in Economics, núm. DP 101, Falmer, University of Sussex, pp. 1-29. 
Frías, Sonia M. (2011), "Hostigamiento, acoso sexual y discriminación laboral por embarazo en México", Revista Mexicana de Sociología, vol. 73, núm. 2, pp. 329-365.

Friedland, Nehemiah, Shlomo Maital y Aryen Rutenberg (1987), "A Simulation Study of Income Tax Evasion”, Journal of Public Economics, vol. 10, pp. 107-16.

Graetz, Michael J. y Louis L. Wilde (1985), "The Economics of Tax Compliance: Fact and Fantasy", National Tax Journal, vol. 38, núm. 547, pp. 355-63.

Grown, Caren e Imraan Valodia (2007), "The Inequality Edition”, Tax Justice Focus, vol. 3, núm. $1<$ http:/ / www.taxjustice.net/cms/upload/pdf/TJF_31_final.pdf $>$.

Guala, Francesco (2005), The Methodology of Experimental Economics, Nueva York, Cambridge University Press.

Hessing, Dick J. (1992), "Does Deterrence Deter? Measuring the Effect of Deterrence in Field Studies and Experimental Studies”, en Joel Slemrod (coord.), Why People Pay Taxes?, Ann Arbor, University of Michigan Press.

Hoyos, Alejandro y Hugo Nopo (2010), "Evolution of Gender Gaps in Latin America at the Turn of the Twentieth Century", IDB Working Paper Series, núm. 176, Washington, Inter-American Development Bank.

Huber, Evelyne (2005), "Gendered Implications of Tax Reform in Latin America: Argentine, Chile, Costa Rica, and Jamaica”, documento de trabajo, Ginebra, UNRISD Project on Gender and Social Policy, United Nations Research Institute for Social Development.

INEGI (2013), Encuesta Nacional de Ocupación y Empleo. Tercer trimestre, 2013, México, Instituto Nacional de Estadística y Geografía <www.inegi.org.mx>.

Juan, Ana de, Miguel A. Lasheras y Rafaela Mayo (1994), "Voluntary Tax Compliance Behavior of Spanish Income Taxpayers”, Public Finance, vol. 49, suplemento, pp. 90-105.

Mehra, Rekha y Sarah Gammage (1999), "Trends, Countertrends, and Gaps in Women's Employment”, World Development, vol. 27, núm. 3, pp. 533-550.

Martínez, Irma y Gloria J. Acevedo Flores (2004), "La brecha salarial en México con enfoque género, capital humano, discriminación y selección muestral", Ciencia, UANL, vol. 7, núm. 1, pp. 66-71.

Oaxaca, Ronald (2007), "The Challenge of Measuring Labor Market Discrimination against Women", Swedish Economic Policy Review, vol. 14, pp. 199-231.

Pagán, José y Susana Sánchez (2000), “Gender Differences in Labor Market Decisions: Evidence from Rural Mexico”, Economic Development and Cultural Change, vol. 48, pp. 619-637.

Plott, Charles R. (1987), "Dimensions of Parallelism: Some Policy Applications of Experimental Methods", en Alvin E. Roth (coord.), Laboratory Experimentation in Economics: Six Points of View, Nueva York, Cambridge University Press, pp.197-229.

Popli, Gurleen K. (2012), "Gender Wage Differentials in Mexico: A Distribu- 
tional Approach", Journal of the Royal Statistical Society: Series A (Statistics in Society), vol. 17, núm. 2, pp. 295-319.

Poterba, James M. (1987), “Tax Evasion and Capital Gains Taxation”, American Economic Review, vol. 77, pp. 234-39.

Slemrod, Joel (1985), "An Empirical Test for Tax Evasion”, The Review of Economics and Statistics, vol. 67, pp.232-38.

Smith, Vernon L. (1982), "Microeconomic Systems as an Experimental Science”, American Economic Review, vol. 72, pp. 923-955.

Stotsky, Janet (2005), "Sesgos de género en los sistemas tributarios”, documento, Madrid, Instituto de Estudios Fiscales.

Suden, Annika y Brian Surette (1998), "Gender Differences in the Allocation of Assets in Retirement Savings Plans", American Economic Review, vol. 88, núm. 2, pp. 207-11.

Torgler, Benno y Neven T. Valev (2006), "Women and Illegal Activities: Gender Differences and Women's Willingness to Comply Over Time", Andrew Young School of Policy Studies Research Paper, núm. 06-56, Atlanta, Georgia State University.

Yitzhaki, Shlomo (1974), "A Note on Income Tax Evasion: A Theoretical Analysis", Journal of Public Economics, vol. 3, pp. 201-02.

\section{Acerca de la autora}

Laura Sour realizó sus estudios de licenciatura y maestría en Economía en el Instituto Tecnológico Autónomo de México (ITAM). Posteriormente obtuvo la maestría y el doctorado en Políticas Públicas en la Irving B. Harris School de la Universidad de Chicago. Ha sido profesora e investigadora del Centro de Investigación y Docencia Económicas (CIDE) -donde además coordinó el Programa de Presupuesto y Gasto Público- y del ITAM. Dentro de sus líneas de investigación destacan los temas de presupuesto y gasto público, contabilidad gubernamental, finanzas públicas, descentralización, cumplimiento del pago de impuestos y economía experimental. Entre sus publicaciones se encuentran: "The Flypaper Effect in Mexican Local Governments", Estudios Económicos, vol. 28, núm. 1, 2013; "Un repaso de los conceptos sobre capacidad y esfuerzo fiscal, y su aplicación en los gobiernos locales mexicanos”, Estudios Demográficos y Urbanos, vol. 23, núm. 2 (68), pp. 271-297, 2008; disponible en <redalyc.org/articulo.oa?id=31223203>. Actualmente es profesora e investigadora en la Facultad de Economía y Negocios de la Universidad Anáhuac México Norte. Es investigadora nivel I en el Sistema Nacional de Investigadores (SNI). 
